\title{
Pro-Oxidant and Anti-Oxidant Status in Patients of Psoriasis with Relation to Smoking and Alcoholism
}

\author{
P. K. Kute ${ }^{1}$, M. G. Muddeshwar², A. R. Sonare ${ }^{3}$ \\ ${ }^{1}$ Assistant Professor, Department of Biochemistry, Jawaharlal Nehru Medical College, Wardha, Maharashtra, India. \\ 2Ex-Professor, Department of Biochemistry, Government Medical College, Nagpur, Maharashtra, India. \\ ${ }^{3}$ Consultant Biochemist, Vimtas Laboratories, Hyderabad, Telangana, India.
}

\section{ABSTRACT}

\section{BACKGROUND}

Psoriasis is a chronic inflammatory skin disease. Prevalence of Psoriasis is approximately more than $2 \%$ of general population, According to various studies, genetic susceptibility and environmental factors have been suggested to play an etiological role in psoriasis. The exact etiological factor for psoriasis is still unknown. Recently it is suggested that imbalance between antioxidants and prooxidants may be involved in the pathogenesis of this disease.

\section{METHODS}

This was a comparative cross-sectional study. Diagnosed cases of Psoriasis were taken; out of these 16 were smokers and 12 were alcoholic. 50 age and sex matched healthy normal subjects as controls were also selected for study. In this study, we measured antioxidant enzymes i.e. superoxide dismutase (SOD) and glutathione peroxidase (GPX) as markers of the antioxidant status and malondialdehyde as the marker of pro-oxidant status with relation to lifestyle factors i.e. smoking and alcoholism.

\section{RESULTS}

The mean levels antioxidant enzymes SOD and GPx were $128.14 \pm 27.50 \mathrm{U} / \mathrm{ml}$ and $3.51 \pm 1.15 \mathrm{U} / \mathrm{ml}$ in cases respectively and $209.54 \pm 37.80 \mathrm{U} / \mathrm{ml}$ and $7.17 \pm 1.71$ $\mathrm{U} / \mathrm{ml}$ in controls respectively $(\mathrm{p}<0.0001)$; Malondialdehyde levels were $3.43 \pm 1.10$ $\mathrm{nmol} / \mathrm{ml}$ in cases and $1.69 \pm 0.10 \mathrm{nmol} / \mathrm{ml}$ in controls $(\mathrm{p}<0.0001)$. Moreover, levels of antioxidant enzymes were significantly decreased and malondialdehyde level was significantly increased in cases with smoking and alcohol habits compared to cases without smoking and alcoholism. This change indicates disruption of antioxidant and pro-oxidant status in psoriasis.

\section{CONCLUSIONS}

Oxidative stress can be a causative element in psoriasis, and by modifying the lifestyle factors the severity of psoriasis can be lowered. Increase in lipid peroxidation and decrease in antioxidants levels can be considered as a feature of psoriasis. It may be suggested that to inactivate the effects of free radicals and for stabilization of the cell membranes and for prevention of further epidermal destruction, an antioxidant supplementation should be recommended. During psoriasis treatment smoking and alcohol should be taken into consideration as prevention from bad effects of smoking and alcohol may help to improve the clinical condition of patient.

\section{KEY WORDS}

Psoriasis, Oxidative Stress, Smoking, Alcoholism
Corresponding Author: Dr. Prakash Keshaorao Kute, Assistant Professor, Department of Biochemistry, Jawaharlal Nehru Medical Collge, Sawangi (Meghe), Wardha-442107, Maharashtra, India.

E-mail: prakashkute@gmail.com

DOI: $10.14260 / \mathrm{jemds} / 2019 / 582$

Financial or Other Competing Interests: None.

How to Cite This Article:

Kute PK, Muddeshwar MG, Sonare AR. Prooxidant and anti-oxidant status in patients of psoriasis with relation to smoking and alcoholism. J. Evolution Med. Dent. Sci. 2019;8(34):2677-2680, $10.14260 / \mathrm{jemds} / 2019 / 582$

Submission 29-06-2019,

Peer Review 10-08-2019,

Acceptance 16-08-2019,

Published 26-08-2019. 


\section{BACKGROUND}

Psoriasis is a chronic, inflammatory and proliferative disease of skin. The lesions of psoriasis are red, scaly, sharply demarcated, indurated plaques presenting particularly over extensor surface and scalp.[1] Prevalence of Psoriasis is approximately more than $2 \%$ of general population, [2] According to various studies, genetic susceptibility and environmental factors have been suggested to play an etiological role in psoriasis. Among the causative factors metabolic abnormalities, systemic infections, stress, trauma, drugs, alcohol consumption and smoking are more common. Oxidative stress affects skin due to production of reactive oxygen species (ROS) in keratinocytes.[3] Oxidative injury lead to some biochemical changes in keratinocytes mediated through ROS.[4] Smoking and alcohol overconsumption are the most significant causative factors in certain health problems. Earlier studies have shown that there is an association between cigarette smoking, alcohol consumption, and psoriasis. Smoking appears to be one of the important factors in the initiation of psoriasis. Alcohol consumption worsens pre-existing condition. Smoking could influence psoriasis via different mechanisms; mainly by increasing the oxidative stress.[5] The free radicals initiate oxidation of PUFA resulting in formation of lipid peroxidation product such as Malondialdehyde (MDA).[1] Under physiological conditions free radical injuries are defended by a complex antioxidant system, i.e. antioxidant enzymes and antioxidant molecules present in plasma. The concentration of every antioxidant enzymes in biological fluids contributes to its in vivo antioxidant capacity.[6] In this study, a pro-oxidant Malondialdehyde (MDA) and antioxidant enzymes Superoxide dismutase (SOD) and Glutathione peroxidase (GPx) were measured to know the oxidative and antioxidative Status in patients of psoriasis with relation to smoking habits and alcoholism.

\section{METHODS}

\section{Study Subjects}

This was a cross-sectional study with comparison groups. The study protocol was approved by the Ethical Committee of the Institute. Informed written consent was obtained from all the study subjects enrolled in the study. 50 diagnosed cases of Psoriasis were taken for the study, sample size was calculated by using formula,[7] out of 50 cases 16 were smoker and 12 were alcoholic and 50 age and sex matched healthy normal subjects as controls were also selected for study. Average age of cases was $42.9 \pm 7.3$ and average age of control was $42.4 \pm 9.4$. The diagnosis of patients was done by clinical features like itching, erythema, thickening and scaling of the skin and Auspitz sign, all the study subjects were examined and investigated according to predesigned proforma. Information regarding sociodemographic factors, alcohol consumption, smoking habits, and personal medical history was obtained from psoriatic patients and controls using questionnaire. The cases treated with topical agents such as dithranol, corticosteroids, vitamin D analogues, during the six month period were included in the study. Patients with IHD, renal disorders, obstructive liver disease, hypothyroidism, diabetes mellitus, any chronic inflammatory disease and any other skin disorder were excluded from the study. Cases taking systemic drug therapy of retinoids, cyclosporine, beta blockers and thiazides in the recent 6 months were excluded from the study.

\section{Collection and Processing of Samples}

About $5 \mathrm{ml}$ of venous blood sample were collected. Malondialdehyde, Glutathione peroxidase and superoxide dismutase were estimated on the same day. Malondialdehyde was measured by Kei Satoh method,[8] Glutathione peroxidase was measured by Paglia and Valentine method[9] and superoxide dismutase by Enzymatic method.[10]

\section{Statistical Analysis}

SOD, GPx, and MDA levels were compared between cases and control by performing unpaired t-test. SOD, GPx, and MDA levels of cases were also compared with cases having smoking habit and cases without smoking habit and also cases having alcohol habit and without alcohol habit by performing unpaired t-test. p-Value $<0.05$ was considered as statistically significant, Statistical software STATA version 10.0 was used for statistical analysis.

\section{RESULTS}

\begin{tabular}{|c|c|c|c|}
\hline Parameter & Cases (N=50) & Controls (N=50) & P \\
\hline SOD $(\mathrm{U} / \mathrm{ml})$ & $128.14 \pm 27.50$ & $209.54 \pm 37.80$ & $\mathrm{p}<0.0001$ \\
\hline $\mathrm{GPx}(\mathrm{U} / \mathrm{ml})$ & $3.51 \pm 1.15$ & $7.17 \pm 1.71$ & $\mathrm{p}<0.0001$ \\
\hline MDA $(\mathrm{nmol} / \mathrm{ml})$ & $3.43 \pm 1.10$ & $1.69 \pm 0.10$ & $\mathrm{p}<0.0001$ \\
\hline
\end{tabular}

Table 1. Pro-oxidant and Antioxidant Status in Cases and Controls

\begin{tabular}{|c|c|c|c|}
\hline Parameter (Unit) & Group I (N=16) & Group II (N=34) & P \\
\hline SOD (U/ml) & $112.8 \pm 18.72$ & $135.35 \pm 28.21$ & $<0.0056$ \\
\hline $\mathrm{GPx}(\mathrm{U} / \mathrm{ml})$ & $2.64 \pm 0.80$ & $3.92 \pm 1.06$ & $<0.0001$ \\
\hline MDA (nmol/ml) & $4.30 \pm 0.91$ & $3.02 \pm 0.78$ & $<0.0001$ \\
\hline Table 2. Pro-Oxidant and Antioxidant Status in Psoriasis Cases with \\
Smoking Habits (Group I) and Psoriasis Cases without Smoking \\
Habits (Group II)
\end{tabular}

\begin{tabular}{|c|c|c|c|}
\hline Parameter (Unit) & Group I (N=12) & Group II (N=38) & P \\
\hline SOD $(\mathrm{U} / \mathrm{ml})$ & $114.0 \pm 18.44$ & $132.60 \pm 28.55$ & $<0.0397$ \\
\hline GPx $(\mathrm{U} / \mathrm{ml})$ & $2.87 \pm 1.05$ & $3.71 \pm 1.11$ & $<0.0264$ \\
\hline $\mathrm{MDA}(\mathrm{nmol} / \mathrm{ml})$ & $4.13 \pm 0.98$ & $3.21 \pm 0.15$ & $<0.0051$ \\
\hline
\end{tabular}

Table 3. Pro-Oxidant and Antioxidants in Psoriasis Cases with Alcohol Habits (Group I) and Psoriasis Cases without Alcohol Habits (Group II)

\section{DISCUSSION}

Recently it has been suggested that increased ROS production and diminished function of antioxidant systems may be involved in the pathogenesis of psoriasis.[11] Keratinocytes are the major source of ROS generated in healthy skin. With psoriatic lesions ROS are generated by both keratinocytes and activated inflammatory cells, mostly neutrophils.[12] When natural antioxidant defence systems are overpowered by a prolonged production of ROS, the resulting oxygen free radicals damage the proteins, lipids and DNA.[13] It has been suggested by certain workers that increased production of ROS and inefficient function of anti-oxidant systems may be involved in the pathogenesis of psoriasis.[14]

In the present study we tried to explore whether the levels of serum MDA are enhanced in psoriasis patients or otherwise compared to healthy controls. It was found that 
MDA was significantly increased in cases of Psoriasis as compared to controls.

It is well established that the ROS induced oxidation of polyunsaturated fatty acids in biological system results in the enhancement of lipid peroxidation and MDA being one of its product, has been considered as a biomarker of lipid peroxidation.[15] These finding are in agreement with the observation of Gupta $M$ et al[16] and Gornicki $A,[17]$ who noticed the elevated in serum MDA levels in psoriasis. Samuel $\mathrm{V}$ et $\mathrm{al}^{[1]}$ and Kadam $\mathrm{D}$ et al[11] have noticed concomitant increase in MDA with the severity of psoriasis. ROS may be produced during the inflammatory process, in psoriasis, affecting primarily lipid metabolism of cells. Further, ROS that are produced by lipid peroxidation may activate phospholipase $\mathrm{A}_{2}$ and thus causing peroxidation of many mediators which finally metabolized to MDA.[18],[19]

Our study has also revealed a significant lowering in plasma activity of SOD and GPx in psoriasis patients as compare to the controls.

SOD, an antioxidant enzyme, accelerates the dismutation of the toxic superoxide radicals produced during the oxidative energy processes into the less harmful molecules, hydrogen peroxide and molecular oxygen. It may be suggested that the lowering in SOD activity may result into more accumulation of superoxide anion radicals in neutrophils and such neutrophils may also get accumulated in psoriatic lesions where they are responsible for abundant superoxide production during the phagocytic reaction and also for the systemic activation of circulating neutrophils in psoriatic patients. ${ }^{[18]}$

Cellular GPx enzyme which changes the hydroperoxide group to the much less toxic hydroxyl moiety, this enzymes is particularly involved in the detoxification of the cell in oxygen radicals and it seems that the failure of this mechanism may account for the lipid peroxidation of the RBC membrane.[20]

Our findings are consistent with study done by Gupta M et al,[16] showing significant lowering in plasma SOD in psoriasis. Drewa G et al,[21] found that SOD activity is lowered to the extent of $20 \%$ as compare to that in controls. Topical treatment improved the patient's clinical condition, resulting in simultaneous increase of enzyme activity. During present study we could also record the significantly decrease activity of Gpx in psoriasis patients as compare to the controls. Our findings are consistent with the finding of Gupta $M$ et al[16] and P. Rocha-Pereira et al..[20]

It may be postulated that decreased SOD and Gpx activity could be responsible for the increased superoxide anion production during the psoriatic process in the skin as well as for the activation of peripheral neutrophils. Increased superoxide anion production might also affect lipid peroxidation, as reflected by increased MDA levels In our present study, antioxidants enzymes viz. superoxide dismutase and glutathione peroxidase concentration in patients of psoriasis with smoking habit were recorded to be profoundly low compared to psoriasis patients. Serum MDA levels were found to be more significantly elevated in psoriasis cases with smoking habits as compared to psoriatic patients. Naldi et al[22] showed that the risk for plaque psoriasis was higher with current smokers compare to exsmokers. Setty AR et al[23] while examining incident of psoriasis found a significantly positive co-relationship between the numbers of cigarettes smoked per day and development of psoriasis, especially in women. It has also been documented in the past that smoking worsens psoriasis and patients with psoriasis who smoke tend to be less responsive to treatment. ${ }^{[24]}$

In the present study we also tried to find out whether alcohol consumption has any added effect on oxidative stress in patients of psoriasis. Significantly lowered levels of SOD and Gpx have observed in plasma of psoriasis cases with alcohol habit as compare to the psoriasis without alcohol habit. However highly raised levels of MDA were found in cases with alcohol habit. Over the past decade a possible association between alcohol consumption and psoriasis has been discussed and several large epidemiological studies have been performed. The evaluated data points out a positive link between alcohol consumption and psoriasis. It appears that high consumption of alcohol may trigger, exacerbate and influence the severity and the course of psoriasis.[5] Alcohol can, not only trigger psoriasis, but also influences the course and nature of the disease as heavy drinkers were shown to be have more severe, extensive, and inflamed disease.[5] Poikolainen $\mathrm{K}$ et al[25] and Zimmerman $\mathrm{GM}^{[26]}$ documented that psoriasis is more frequent in alcoholics than in controls. The fact that heavy drinkers usually tend to pay less attention to healthy nutrition (rich in natural antioxidants) may be responsible for this.[27],[28] Certain investigators suggest that alcohol affects psoriasis mainly by altering the immune system. Proposed mechanisms have included suppression of cell-mediated immunity, ${ }^{[29]}$ up-regulation of pro-inflammatory cytokines, ${ }^{[30]}$ and enhancement of mitogen-driven lymphocyte proliferation.[31]

Earlier Smith KE et al[32] have also shown that alcohol stimulates the release of histamine and skin lesions can aggravate as a consequence. Smoking and alcohol are the major factors affecting both the antioxidant system and the production of free radicals and reactive oxygen species.[33],[34] Our study could clearly demonstrate that smoking and alcohol causes further disturbance in oxidant/antioxidant status and dyslipidaemia of psoriasis. The antioxidant system of these patients may possibly be in a depressed state or may be overloaded due to oxidative stress caused by smoking and/or alcohol. Therefore it may be stated that the patients with psoriasis who are also alcoholic and/or smokers can require a long period of treatment, hence while designing a treatment strategy these risk factors need to be considered. Further prevention of alcohol and smoking habits may certainly lower the severity of psoriasis, and may help to alleviate their disease and may improve may the quality of life.

\section{CONCLUSIONS}

Compared to controls, psoriasis cases had significantly higher levels of serum MDA with diminished plasma SOD and Gpx. Increase in lipid peroxidation and decrease in antioxidant levels can be considered as a feature of psoriasis. It may be suggested that to inactivate the effects of free radicals and for stabilization of the cell membranes and for prevention of further epidermal destruction, an antioxidant supplementation should be recommended. During psoriasis 
treatment smoking and alcohol should be taken into consideration as prevention from bad effects of smoking and alcohol may help to improve the clinical condition of patient.

\section{REFERENCES}

[1] Samuel TV, Murari K. Potential role of oxidative stress and antioxidant deficiency in pathogenesis of psoriasis. Int J Pharm Bio Sci 2013;4(3):B1039-B44.

[2] Mallbris L, Granath F, Hamsten A, et al. Psoriasis is associated with lipid abnormalities at the onset of skin disease. J Am Acad Dermatol 2006;54(4):614-21.

[3] Maccrarsone M, Catani MV, Iraci S, et al. A survey of reactive oxygen species and their role in dermatology. Eur Acard Dermatol Veneral 1997;8(3):185-202.

[4] Trouba KJ, Hamadeh HK, Amin RP, et al. Oxidative stress and its role in skin disease. Antioxid Redox Signal 2002;4(4):665-73.

[5] Luty-Frąckiewicz A, Markiewicz-Górka I., Januszewska L. Influence of smoking and alcohol consumption on total antioxidant status in patients with psoriasis. Adv Clin Exp Med 2006;15(3):463-9.

[6] Sies H. Oxidative stress: oxidants and antioxidants. Exp Physiol 1997;82(2):291-5.

[7] Charan J, Biswas T. How to calculate sample size for different study designs in medical research? Indian J Psychol Med 2013;35(2):121-6.

[8] Satoh K. Serum lipid peroxide in cerebrovascular disorder determined by a new colorimetric method. Clin Chim Acta 1978;90(1):37-43.

[9] Glutathion peroxidase reagent set (RANSEL) (kit insert). Crumlin (United Kingdom): RANDOX Laboratories Ltd.

[10] Superoxide dismutase reagent set (RANSOD) (kit insert). Crumlin (United Kingdom): RANDOX Laboratories Ltd.

[11] Kadam DP, Suryakar AN, Ankush RD, et al. Role of oxidative stress in various stages of psoriasis. Indian Journal of Clinical Biochemistry 2010;25(4):388-92.

[12] Pelle E, Mammone T, Maes D, et al. Keratinocytes act as a source of reactive oxygen species by transferring hydrogen peroxide to melanocytes. J Invest Dermatol 2005;124(4):793-7.

[13] Kohen R. Skin antioxidants: their role in aging and in oxidative stress - new approaches for their evaluation. Biomed Pharmacother 1999;53(4):181-92.

[14] Briganti S, Picardo M. Antioxidant activity, lipid peroxidation and skin diseases. What's new? J Eur Acad Dermatol Venereol 2003;17(6):663-9.

[15] Lykkesfeldt J. Malondialdehyde as biomarker of oxidative damage to lipids caused by smoking. Clin Chim Acta 2007;380(1-2):50-8.

[16] Gupta M, Chari S, Borkar M, et al. Dyslipidaemia and oxidative stress in patients of psoriasis. Biomedical Research 2011;22(2):221-4.

[17] Gornicki A, Gutsze A. Erythrocyte membrane fluidity changes in psoriasis: an EPR study. J Dermatological Sciences 2001;27(1):27-30.

[18] Baz K, Cimen MY, Kokturk A, et al. Oxidant / anti-oxidant status in patients with psoriasis. Yonsei Medical Journal 2003;44(6):987-90.
[19] Yildirim M, Inaloz HS, Baysal V, et al. The role of oxidants and antioxidants in psoriasis. J Eur Acad Dermatol Venereol 2003;17(1):34-6.

[20] Rocha-Pereira P, Santos-Silva A, Rebelo I, et al. Erythrocyte damage in mild and severe psoriasis. British J of Dermatol 2004;150(2):232-44.

[21] Drewa G, Krzyzynska-Malinowska E, Wozniak A, et al. Activity of superoxide dismutase and catalase and the level of lipid peroxidation product reactive with TBA in patients with psoriasis. Med Sci Monit 2002;8(8):BR33843.

[22] Naldi L, Chatenoud L, Linder D, et al. Cigarette smoking, body mass index and stressful life events as risk factors for psoriasis: results from an Italian case-control study. J Invest Dermatol 2005;125(1):61-7.

[23] Setty AR, Curhan G, Choi HK. Smoking and the risk of psoriasis in women: Nurses' Health Study IL Am J Med 2007;120(11):953-9.

[24] Armstrong AW, Armstrong EJ, Fuller EN, et al. Smoking and pathogenesis of psoriasis: a review of oxidative, inflammatory and genetic mechanisms. Br J Dermatol 2011;165(6):1162-8.

[25] Poikolainen K, Reunala T, Karvonen J. Smoking, alcohol and life events related to psoriasis among women. Br J Dermatol 1994;130(4):473-7.

[26] Zimmerman GM. Alcohol and psoriasis: a double burden. Arch Dermatol 1999;135(12):1541-2.

[27] Ringstad J, Knutsen SF, Nilssen OR, et al. Comparative study of serum selenium and vitamin $\mathrm{E}$ levels in a population of male risk drinkers and abstainers. A population based matched-pair study. Biol Trace Elem Res 1993;36(1):65-71.

[28] Gueguen S, Pirollet P, Leroy P, et al. Changes in serum retinol, alpha-tocopherol, vitamin $\mathrm{C}$, carotenoids, zinc and selenium after micronutrient supplementation during alcohol rehabilitation. J Am Coll Nutr 2003;22(4):303-10.

[29] Nordmann R, Rouach H. Alcohol and free radicals: from basic research to clinical prospects. Bull Acad Natl Med 1995;179(9):1839-53.

[30] Higuchi H, Kurose I, Kato S, et al. Ethanol-induced apoptosis and oxidative stress in hepatocytes. Alcohol Clin Exp Res 1996;29(Suppl 9):340A-6A.

[31] Schopf RE, Ockenfelds HM, Morsches B. Ethanol enhances the mitogen-driven lymphocyte proliferation in patients with psoriasis. Acta Derm Venereol (Stockh) 1996;76(4):260-3.

[32] Smith KE, Fenske NA. Cutaneous manifestations of alcohol abuse. J Am Acad Dermatol 2000;43(1 Pt 1):1-16, quiz 16-8.

[33] Papas AM. Determinants of antioxidant status in humans. Lipids 1996;(Suppl 31):S77-S82.

[34] Marangon $\mathrm{K}$, Herbeth $\mathrm{B}$, Lecomte $\mathrm{E}$, et al. Diet, antioxidant status and smoking habits in French men. Am J Clin Nutr 1998;67(2):231-9. 